Ici les formules anciennes reprennent l'avantage. On voit apparaître la supériorité des formules de Lévy et de Reynolds, et l'infériorité de celle de Flamant, pour les gros diamètres.

Au siphon de Saint-Paul, sur le canal de Verdon, un tuyau de 1,75 de diamètre dont la pente est de 0,001 débite jusqu'à $3 \mathrm{~ms}^{3}$ d'eau par seconde; le diamètre a été calculé par la formule de Prony pour un débit de 2500 litres seulement.

Les formules accusent pour $D=1^{\mathrm{m}} 75$ et $J=0^{\mathrm{m}} 001$ :

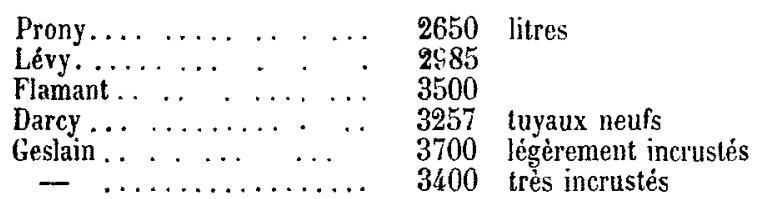

Cette expérience fait nettement ressortir l'exactitude de la formule de Lévy pour les gros diamètres.

Les réservoirs de chasse qui servent au nettoyage des égouts de Paris, sont alimentés par des tubes en fer de 0,005 et 0,004 de diamètre, qui doivent fournir uniformément ro $\mathrm{ms}^{3}$ par vingt-quatre heures ou ol i 6 à la seconde. Ce résultat est sensiblement acquis. Les tubes de 0,005 s'emploient pour les pressions comprises entre 5 et $45 \mathrm{~ms}$, ceux de 0,004 pour les pressions pius élevées.

Dans les tubes de 0,005 la vitesse est de $6 \mathrm{~ms}$ par seconde; la perte de charge se rapproche de $15 \mathrm{~ms}$, car les tubes ont $3 \mathrm{~ms}$ de long pour $45 \mathrm{~ms}$ de pression sur la conduite, 2,60 pour $40 \mathrm{~ms}$ et $2 \mathrm{~ms}$ seulement pour $30 \mathrm{~ms}$.

Les diverses formules donnent comme perte de charge par mètre pour $D=0^{\mathrm{m}} .005$ et $Q=\mathrm{o}_{1}^{1}$ I 6 :

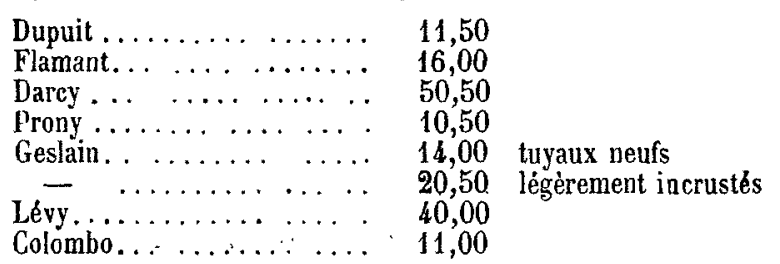

Ces chiffres marquent la supériorité des formules de Flamant et de Geslain pour les petits diamètres.

Le tableau suivant donne les débits en litres par seconde correspondant à une perte de charge de 5 millimètres par mètre pour des tuyaux de $0^{m} 500, o^{m} 800$ et I mètre de diamètre :

\begin{tabular}{|c|c|c|}
\hline & $0^{\mathrm{m}} 500$ & $0 \operatorname{son} 800$ \\
\hline 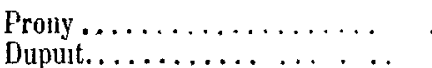 & $\begin{array}{l}260 \\
251\end{array}$ & $\begin{array}{l}839 \\
810\end{array}$ \\
\hline Darcy tuyaux neufs. & 300 & 988 \\
\hline Darcy t tuyaux incrustés & 212 & 695 \\
\hline 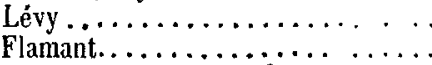 & $\begin{array}{l}225 \\
316\end{array}$ & $\begin{array}{r}785 \\
1128\end{array}$ \\
\hline 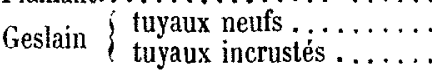 & $\begin{array}{l}320 \\
260\end{array}$ & $\begin{array}{r}1150 \\
925\end{array}$ \\
\hline
\end{tabular}

Conclusion. - Personnellement, nous donnons la préférence à la formule de M. Flamant pour les petits et moyens diamètres jusqu'à I mètre, et à celle de $M$. Lévy pour les diamètres plus élevés.

Néanmoins, nous reconnaissons que pour les diamètres compris entre 0,15 et 0,80 , on obtient des résultats assez approchés avec la plupart des formules anciennes, et celles de Darcy, Manning, Kutter, etc. Les formules de Reynolds se présentent également dans d'assez bonnes conditions sur toute l'échelle des diamètres.

DARIÈS.

\section{Distribution d'Énergie Électrique dans le département de l'Aude}

Distribuer l'énergie électrique au moyen d'une usine génératrice unique, aussi bien pour la force motrice que pour l'éclairage dans un département tout entier, couvrir. ce département d'un réseau de lignes à haute tension, utiliser pour cela une chute d'eau située à 70 kilomètres d'un centre de distribution d'oú partent des dérivations ayant 30 et $4^{\circ}$ kilomètres de longueur, tel était le programme à remplir, tel est le projet qui a été réalisé dans le département de l'Aude.

L'exécution d'une semblable installation correspond à un notable progrès de l'industrie électrique et son fonctionnement depuis trois ans permet de tirer des conclusions de la plus haute importance au point de vue pratique.

Il existe actuellement peu d'installations semblables en' Europe, car le réseau actuel ne comprend pas moins de. 600 kilomètres de canalisations à courant triphasé à haute tension, avec des distances de roo à 130 kilomètres entre l'usine génératrice et le point d'utilisation le plus éloigné. En tout cas, cette installation est la première de ce genre exécutée en France et elle a été conçue et exécutée par: M. Estrade, directeur de la Société méridionale de Trans: port de force, qui a confié la partie électrique et mécanique à la Société d'applications industrielles laquelle a employé exclusivement le matériel électrique Alioth.

Le réseau de distribution fournit le courant électrique plus de cent communes d'importances diverses, mais repreisentant une population globale de plus de 150000 habl: tants.

L'usine génératrice est située dans les Corbières, à l'en trée des Gorges de Saint-Georges, à 2 kilomètres d'Axat Elle utilise une chute de l'Aude ayant un peu plus de roo mètres de hauteur. - Un long feeder part de cettè usine et aboutit après un parcours de 70 kilomètres au: centre de distribution situé à Fabrezan, petit chef-lieu dé canton du département de l'Aude. De ce centre de distribue tion partent trois dérivations principales, allant la premiére à Narbonne, à une distance de 30 kilomètres; la seconde à Carcassonne, à une distance de 35 kilomètres; la troisième à La Nouvelle, à une distance de 30 kilomètres:Ces dérivations principales fournissent l'énergie directement en tous les points placés sur leur passage, mais elles servent surtout à alimenter les centres de distribution dans lesquels la haute tension est réduite en moyenné tension, et c'est de ces centres de distribution secondaires que rayonnent les lignes de distribution proprement dites. - C'est ainsi que Narbonne, centre de distribution, alis mente ses environs : Vinassan, Armissan, Salles et Fleury; que Portel, centre de distribution, alimente Sijean, La Nouvelle, Peyriac de Mer, et que Carcassonne, centre de distribution, alimente les environs de cette ville, Pezens, Moussoulier, Bram, Pennautier, etc.

Quand s'est présentée l'étude d'un semblable projet, il y a plus de six ans (car l'exécution en fut retardée par suite de divers accidents et incidents), le point le plus délicat, comme on peut se le figurer, résidait dans le choix dé la tension. On conçoit aisément que pour des distances aussi grandes, il fallait une tension très élevée, présentant: néanmoins toutes les garanties désirables aussi bien au point de vue des facilités de l'exécution qu'au point de vaé 
de la bonne marche industrielle. L'emploi. du courant triphasé s'imposait sans discussion possible et la fréquence adoptée fut celle de 50 périodes par seconde. Il fallait, de plus, adopter une tension en rapport avec la quantité d'énergie à transporter, et qui permît en outre de placer des transformateurs de puissance assez faible puisque la distribution de l'énergie devait être très divisée. Enfin, il ne faut pas perdre de vue qu'il y a six ans nos connaissances en fait de haute tension étaient beaucoup moins considérables quà l'époque actuelle.

L'usine de Saint-Georges devait pouvoir fournir 3200 kilowatts, mais l'installation première comportait seulement la production et la distribution de la moitié de cette puissance, soit seulement i ooo kilowatts. Dans ces conditions, on a considéré que la tension ne devait pas dépasser 20000 volts. C'est celle que l'on a été conduit à adopter comme permettant de donner les meilleures garanties tant pour le fonctionnement des lignes que pour la bonne construction de transformateurs de puissances relativement faibles qui devaient être prévus.

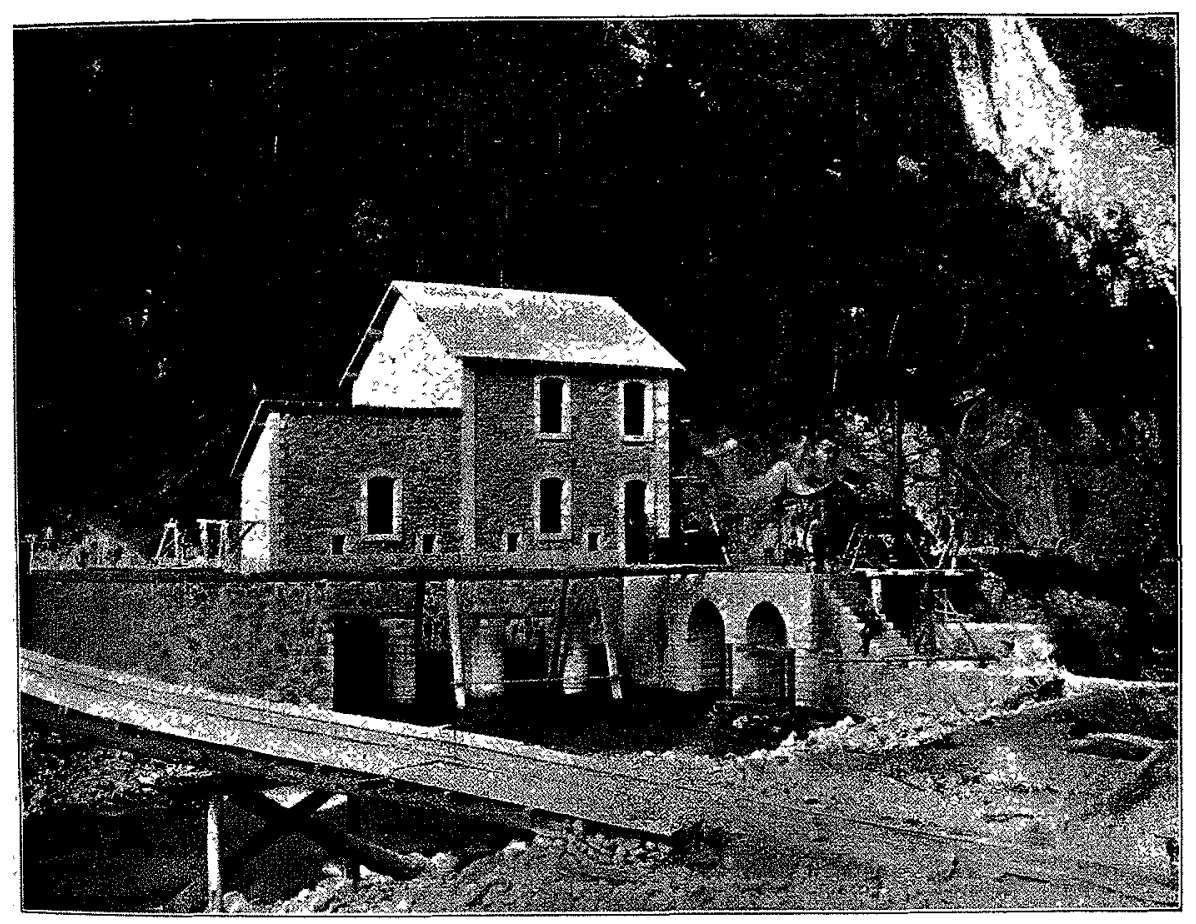

Fig. I - Vue du Barrage et de la prise d'eau pendant la construction. profondeur et y enfoncer des pieux. Comme le barrage est de très faible hauteur, ce mode de fondation peut être acceptable.

Le débit de la rivière d'Aude est assez inégal comme celui de toutes les rivières torrentielles. Pendant dix mois de l'année cependant il n'est pas inférieur à 6 ooo litres, il tombe pendant les deux autres mois à 4300 litres. Malgré cela, en prévision de la réalisation d'un barrage réservoir dans la haute vallée de l'Aude, tous les travaux hydrauliques ont été faits pour un débit de 6 mètres cubes. C'est pour ce débit que le canal d'amenée d'eau à l'usine a été prévu. Ce canal est souterrain sur la plus grande partie de son parcours d'une longueur totale de 5500 mètres : il a été percé sur le flanc de la montagne et les points d'attaque ont été aussi nombreux qu'il était nécessaire pour une exécution rapide des travaux. Lors du forage de cette galerie on a rencontré deux sortes de terrains, des terrains calcaires et des terrains schisteux. Dans les premiers, il a suffi d'un léger béton et d'un ciment lissé par-dessus pour constituer le canal, car les calcaires présentaient toute solidité. Il n'en fut pas de même dans le schiste ardoisé qui est très solide lorsqu'il est à l'abri de l'air, mais qui devient très friable au contact de ce dernier; pour ces parties du souterrain il a donc fallu maçon. ner une voûte surbaissée reposant sur des piédroits.

Dans une partie de son parcours, sur une longueur de I 500 mètres environ, le canal est à air libre et en ciment armé monté sur poteaux de même composition. Dans cette partie la montagne n'offrait pas des apparences de solidité suffisante, et de plus la traversée d'une vallée nécessitait la construction d'un petit ouvrage d'art que l'emploi du ciment armé rendait très facile à exécuter. Cette partie du canal n'a causé jusqu'à présent aucun déboire, deux hivers avec de fortes gelées ont cependant passé sans causer le moindre dégât.

Le souterrain a une section sensiblement rec. tangulaire de 2 mètres sur 2 mètres et une pente uniforme de 1,6 millimètres par mètre; dans la partie du canal en ciment armé on a donné une pente plus forte afin de réduire la section. A environ 200 mètres de l'usine se trouve un déversoir, et immédiatement après est située la chambre de retenue, origine des conduites forcées. Une grille et des vannes sont installées pour chacun des tuyaux sous pression, prévus au nombre de quatre, et dont deux sont actuellement en place. Chaque conduite est en tôle d'acier de I mètre de diamètre, rivée par tronçons de trois mètres de longueur, et elle peut débiter I 600 litres d'eau à la seconde avec une vitesse de 2 mètres.

La chute brute, entre le niveau de l'eau dans la chambre de retenue et le niveau de l'eau dans le canal de fuite, est de $101^{m} 40$. La conduite a environ 160 mètres de longueur. A 12 mètres au-dessus de l'usine, chaque tuyau de un mètre se dédouble en deux autres tuyaux de $0^{m} 70$ de diamètre, alimentant chacun une turbine. Jusqu'à la bifurcation, la conduite est libre et peut glisser sur les piliers en maçonnerie qui la supportent. Pour permettre la dilatation au point de bifurcation, elle est maintenue par des chaises en fer ancrées dans un bloc de maçonnerie de 40 mètres 
cubes; et, afin de compenser les dilatations et contractions du tuyau avec la température, il a été prévu, près de la chambre de retenue, un joint de dilatation composé de deux demi-calottes en tôle d'acier.

Pour éviter les coups d'eau qui se produisent lorsqu'il y a mélange d'air et d'eau, un reniflard, sorte de tube percé de trous qui permettent l'évacuation de l'air, a été pratiqué à la partie supérieure de chaque conduite.

La visite des conduites est assurée par des trous d'homme placés de distance en distance.

La perte de charge est de $\mathrm{I}^{\mathrm{m}} \mathrm{I} O$ dans chaque conduite lorsque le débit atteint I 600 litres et alimente deux des turbines de l'usine génératrice.

\section{USINE HYDRO-ÉLECTRIQUE DE SAINT.GEORGES}

La station gènératrice est composée d'un grand bâtiment rectangulaire flanqué à ses deux extrémités de deux pavillons; la partie centrale de l'usine contient les machines, turbines et alternateurs; les pavillons contiennent les transformateurs élévateurs, les tableaux haute tension, l'atelier de réparation et quelques logements.

Au point de vue des machines, l'usine est prévue pour huit groupes électrogènes turbines et alternateurs de 800 chevaux.

Les turbines, de la Société des Ateliers de construction de Vevey, sont à axe horizontal, elles tournent à 300 tours par minute et sont prévues pour développer normalement 800 chevaux. Ce sont des appareils du genre Pelton. Le distributeur a quatre orifices. Un tiroir cylindrique coulissant sur ce distributeur par l'intermédiaire d'un roulement à billes et commandé par le régulateur automatique de vitesse, ou à- la main, permet une plus ou moins grande introduction de l'eau.

L'axe des turbines est à $3 \mathrm{~m} 50$ au-dessus du niveau de l'eau dans le canal de fuite; ces turbines ne peuvent pas fonctionner noyées. Néanmoins, cette hauteur de chute n'est pas perdue complètement: par suite d'un dispositif spécial qui laisse rentrer l'arr et empêche la turbine de se noyer, il se maintient dans le tuyau d'échappement une colonne d'eau mélangée d'air produisant un vide relatif, et permettant de regagner une partie des $3 \mathrm{~m} 5 \mathrm{o}$ de chute que, sans ce dispositif, il eut été impossible d'utiliser.

Chaque turbine est munie d'un régulateur automatique de vitesse, dont le fonctionnement est des plus satisfaisants. Ce régulateur est à servo-moteur hydraulique, utilisant pour cela l'eau sous pression de la chute, préalablement épurée par son passage à travers un filtre.

La variation de vitesse pour une différence de charge brusque de 5o pour 100 n'est pas supérieure à 4 pour roo, et pour 100 pour 100 elle est inférieure à 10 pour 100 . Par suite de la présence d'un régulateur de pression commandé par le régulateur de vitesse, la surpression dans les conduites n'est pas supériecire à Io pour 100 ; les coups de bélier sont ainsi évités. Le rendement de ces turbines est de 77 pour 100 à pleine charge.

Les turbines attaquent directement les alternateurs au moyen de manchons élastiques faisant volant. Ces machines sont du systhème Alioth et capables de fournir 700 kilowatts sous un $\cos \varphi$.égal à l'unité, ou encore 540 kilowatts avec un décalage de 0,80 en absorbant 800 chevaux. Leur tension est de 2900 volts. Ce sont des machines à fer tournant, à courant triphasé à 50 périodes, tournant à 300 tours et bobinées en étoile. Elles portent leurs excitatrices en bout d'arbre. Le courant d'excitation, sous une tension de 50 à 60 volts, est de 25 ampères environ à pleine charge, soit une dépense d'excitation qui n'est pas supérieure à 0,25 pour roo.

Le rendement de ces machines à pleine charge est de $9^{3}$ pour 100 , et leur chute de tension de 5 pour 100 pour $\cos \varphi=1$ et de 16 pour 100 pour $\cos \varphi=0,80$. Elles pèsent 35 tonnes, dont 12 tonnes pour la partie tournante.

Le courant de chaque alternateur traverse immédiate. ment à la sortie de la machine des coupe-circuits à fusibles en argent placés près des machines, convenablement distancés et séparés les uns des autres. C'est de là que part la canalisation reliant les alternateurs au tableau, cette canali. sation est en câble à haut isolement fixé sur isolateurs en porcelaine.

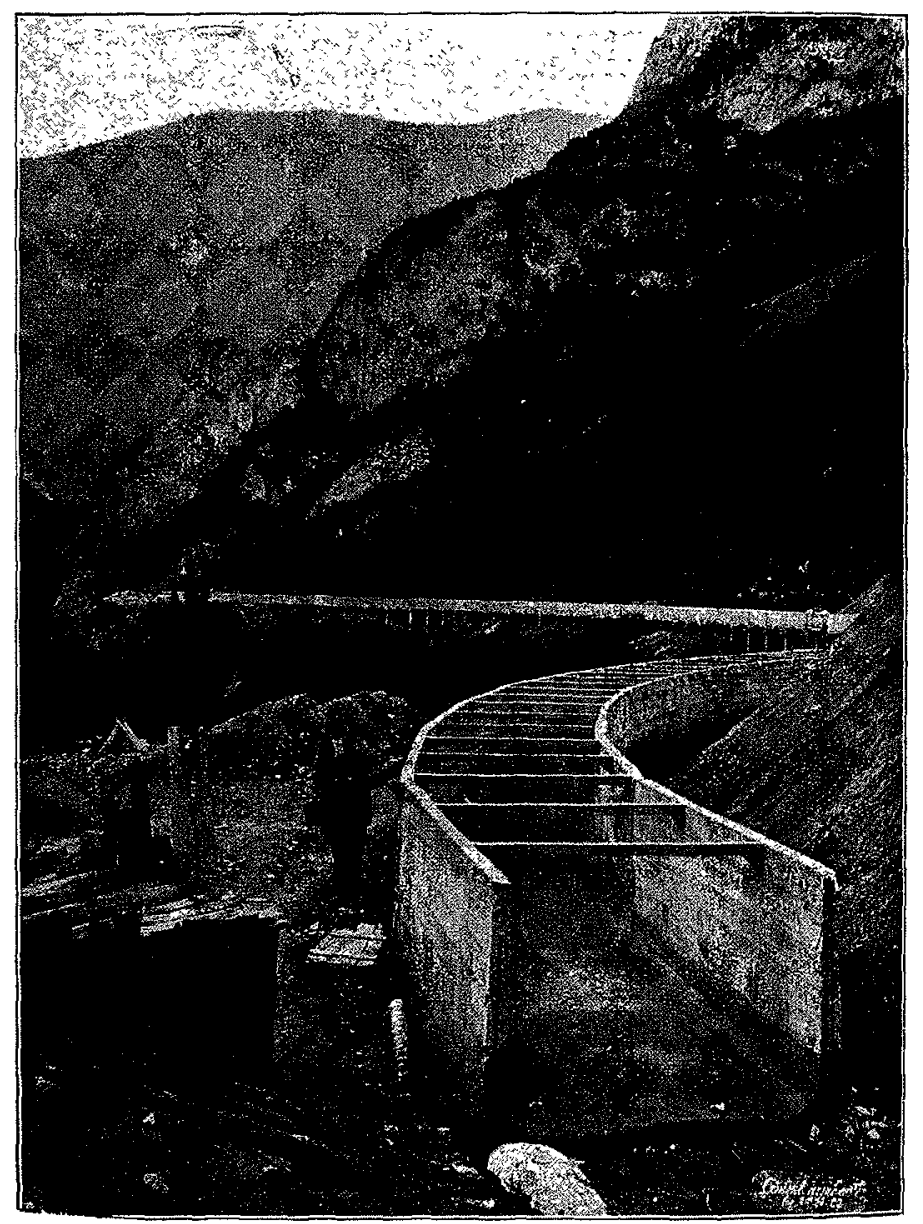

Fig. 2. - Canal d'amenée, partie en ciment armé.

Le tableau des alternateurs comprend un panneau par machine avec tous les appareils usuels: toutes les machines marchent en parallèle sur des barres omnibus. Cette marche en parallèle n'offre d'ailleurs aucune difficulté, chaque tur. bine étant munie de son régulateur automatique de vitesse. Des rhéostats de champ d'alternateurs pouvant être enclanchés les uns avec les autres, permettent, par la manouvivét d'un seul volant, de régler la tension de toutes les machines en service.

C'est sur les barres omnibus des alternateurs que se prend le courant dont la tension doit être portée à 20000 volts par les transformateurs-élévateurs.

Les transformateurs-élévateurs sont du système Alioth à courant monophasé, d'une puissance unitaire de 200 kilo. watts, mais ils sont groupés par trois en étoile, formant ains! un groupe triphasé de 600 kilowatts, correspondant à la 
puissance des alternateurs. Il y a donc actuellement en tout douze transformateurs de 200 kilowatts, à refroidissement naturel par l'air, placés dans une salle spéciale dont la ventilation est effectuée par deux grandes cheminées réservées dans les angles; l'air passant dans la salle des transformateurs vient de la salle des machines, où il est relativement froid et sec.

Le fonctionnement de ces transformateurs à l'air a été toujours très bon; ils avaient d'ailleurs été essayés à 32000 volts, entre les enroulements et la masse pendant une heure. Un seul accident est arrivé à l'un de ces appareils pour une cause absolument imprévue. A l'entrée de lhiver, aux premiers troids, les rats de la montagne ont envahi l'usine et pendant quelques jours on eut toutes les peines du monde à s'en débarrasser': c'est un de ces ron. geurs qui, attiré par la chaleur, vint se cacher dans un transformateur entre les enroulements et la masse, et occasionna un arc dans l'appareil. La réparation fut vite effectuée et on prit des mesures pour empêcher le retour de semblables accidents.

Tous ces transtormateurs fonctionnent en parallele par groupes de trois, et le courant haute tension est conduit à un tableau haute tension placé dans une salle spéciale, au-dessus de celle des transformateurs.

Chaque groupe de transformateurs a, dans la salle des machines, un tableau a la tension des alternateurs, comprenant un interrupteur tripolaire, un ampèremètre et la mancuure des interrupteurs tripolaires haute tension. Cette manœuvre se fait à distance (les interrupteurs haute tension étant placés dans la salle du tableau haute tension), au moyen de l'air comprimé sous une pression de 5 kilogrammes. A cet effet, on a installé un compresseur d'air commandé par un moteur triphasé, alimenté lui-même par le transformateur d'éclairage de l'usine. Un réservoir d'air comprimé, et une luyauterie reliant ce réservoir aux deux tableaux haute et basse tension des transformateurs, complètent l'ensemble de l'installation. Le fonctionnement de tout cet ensemble est très satisfaisant.

Le tableau haute tension comprend les interrupteurs haute tension à manœuvre par l'air comprimé, les fusibles et les barres omnibus 20 ooo volts. Toute la charpente du tableau est en fer et les connexions sont portées sur des isolateurs identiques à ceux de la ligne. Enfin, un groupe de quatre interrupteurs avec un transformateur de mesure dont le secondaire est relié à un voltmètre, permet de mesurer à chaque instant la tension entre fils et entre les fils et la terre. Cette installation rend de grands services car elle permet de s'assurer en marche des défauts qui peuvent se produire sur la ligne et que l'on ne peut constater par les procédés ordinaires. Un plancher isolant porté sur des isolateurs permet d'approcher sans danger de ce tableau. Les sorties de câble haute tension se font par des trous obliques ménagés dans le mur à la sortie de l'usine; et, faisant directement suite à la ligne se trouvent trois parafoudres à corne munis de résistances.

Le tableau haute tension est protégé en plus par des bobines de self composées de spirales faites avec le câble même de la ligne.

\section{LIGNES DE DISTRIBUTION}

Dans un transport d'énergie à grande distance c'est la ligne qui constitue le point faible dusystème. Les lignes de l'Aude furent particulièrement soignées à cet égard.

L'usine génératrice produisant le courant à la tension de 20000 volts, le feeder de 70 kilomètres fut calculé de manière à ce que la perte en énergie fut au maximum de 20 pour 100 lorsque la puissance développée à l'usine génératrice atteindrait 1620 kilowatts avec un facteur de puissance moyen de 0,80 .

Cette perte d'énergie de 20 pour 100 est incontestablement très grande, mais malgré cela le feeder, à lui seul, a exigé 75 tonnes de cuivre, ce qui, au cours élevé auquel on fut obligé de l'acheter il y a quatre ans, représentait déjà une dépense importante. Une perte plus faible eût exigé un poids de cuivre plus considérable et augmenté le capital de premier établissement; or la force motrice était abondante, et de plus il sera facile de diminuer cette perte lorsque les recettes seront suffisantes, en doublant le poids du cuivre,

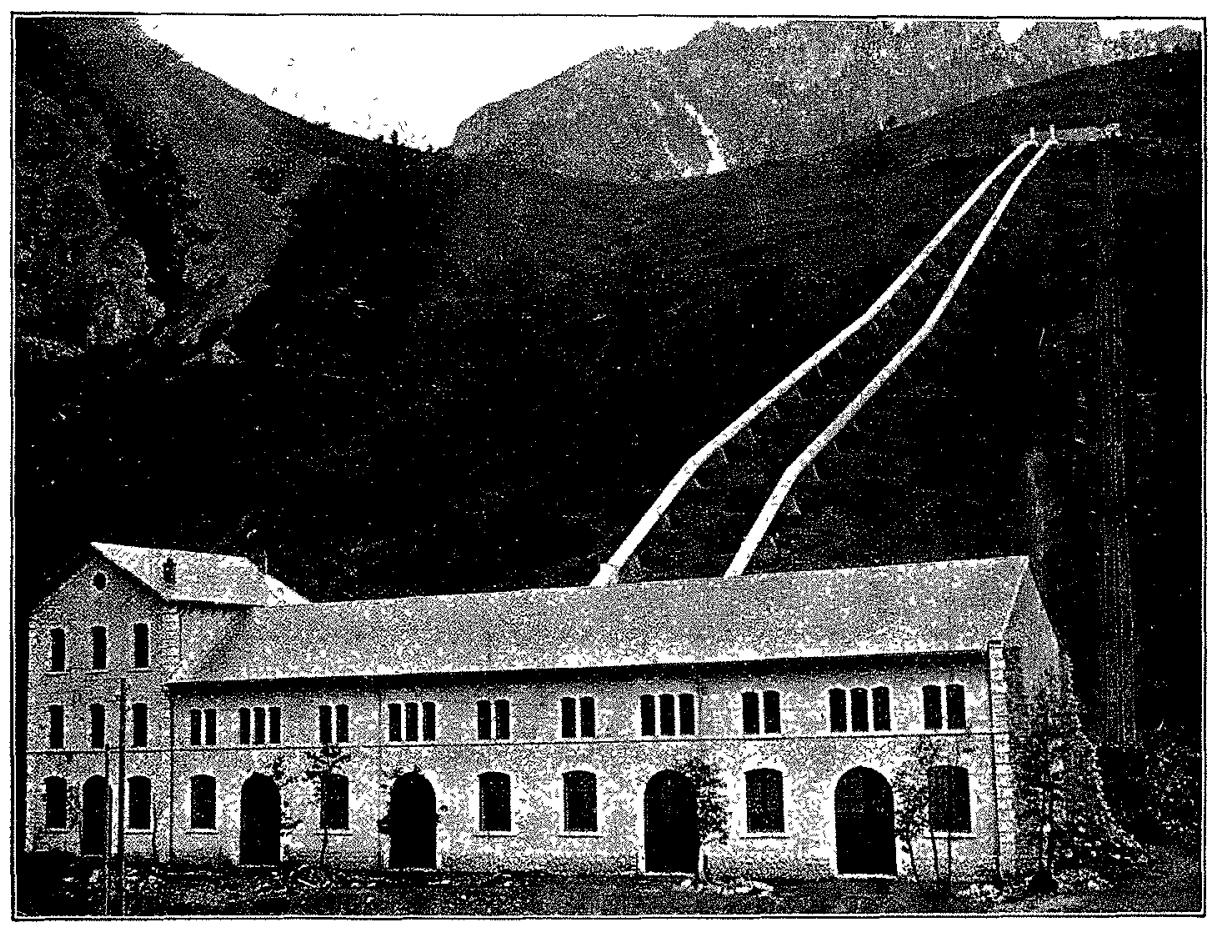

Fig. 3. - Vue de l'Usine et des conduites forcées.

ou, ce qui est mieux, en établissant une seconde ligne parallèle à la première. Pour toutes ces raisons, la perte de 20 pour roo fut considérée comme acceptable, d'autant plus que le décalage de 0,80 était probablement supérieur à celui qui existerait aux heures de pleine charge, car il était convenu que les petits moteurs ne devaient pas fonctionner aux heures d'éclairage. Dans ces conditions, l'expérience a complètement justifié les prévisions, et les difficultés de réglage de tension que l'on pouvait craindre ne se sont pas produites. L'usine génératrice règle donc sa tension d'après les indications des ampèremètres totalisateurs; d'ailleurs une ligne téléphonique relie l'usine génératrice au centre de distribution et permet, le cas échéant, de corriger la tension suivant les indications données par le poste de Fabrezan oú elle est maintenue entre 17000 et 17200 volts.

La section du fil a été facilement calculée avec données suivantes: longueur du feeder, 70 kilomètres; puissance à l'origine, I 620 kilowatts; perte maximum d'énergie, 
20 pour 100 ; tension à l'extrémité du feeder, 17000 volts; décalage des récepteurs à l'extrémité, $\cos \varphi=0,80$.

En écrivant que la perte d'énergie est dépensée en chaleur dans la ligne on a la relation $324000=3 R I^{2}$ dans laquelle $R$ désigne la résistance d'un fil et $I$ le courant qui la traverse: Dans le cas actuel, le courant étant de 54 ampères, on trouve 36,06 ohms comme résistance d'un fil, cette résistance étant calculée à une température de i bo. En tenant compte de la température et de ce que le cuivre du commerce n'est pas pur, on a été amené à poser un câble de 38 millimètres de section, ayant un diamètre de 7,5 millimètres environ.

Quant à la tension à l'usine génératrice, elle a été calculée par la formule :

$$
E^{\prime}=\sqrt{\left(E-R I \sqrt{\overline{3})^{2}+(R I V \overline{3} \operatorname{tg} \varphi)^{2}}\right.}
$$

$E$ ' étant la tension à l'arrivée, $E$ celle au départ. On obtient ainsi comme tension à l'usine génératrice 20240 volts. La perte en volts est donc de 3240 volts, soit environ i 6 p. 100 de la tension au départ.

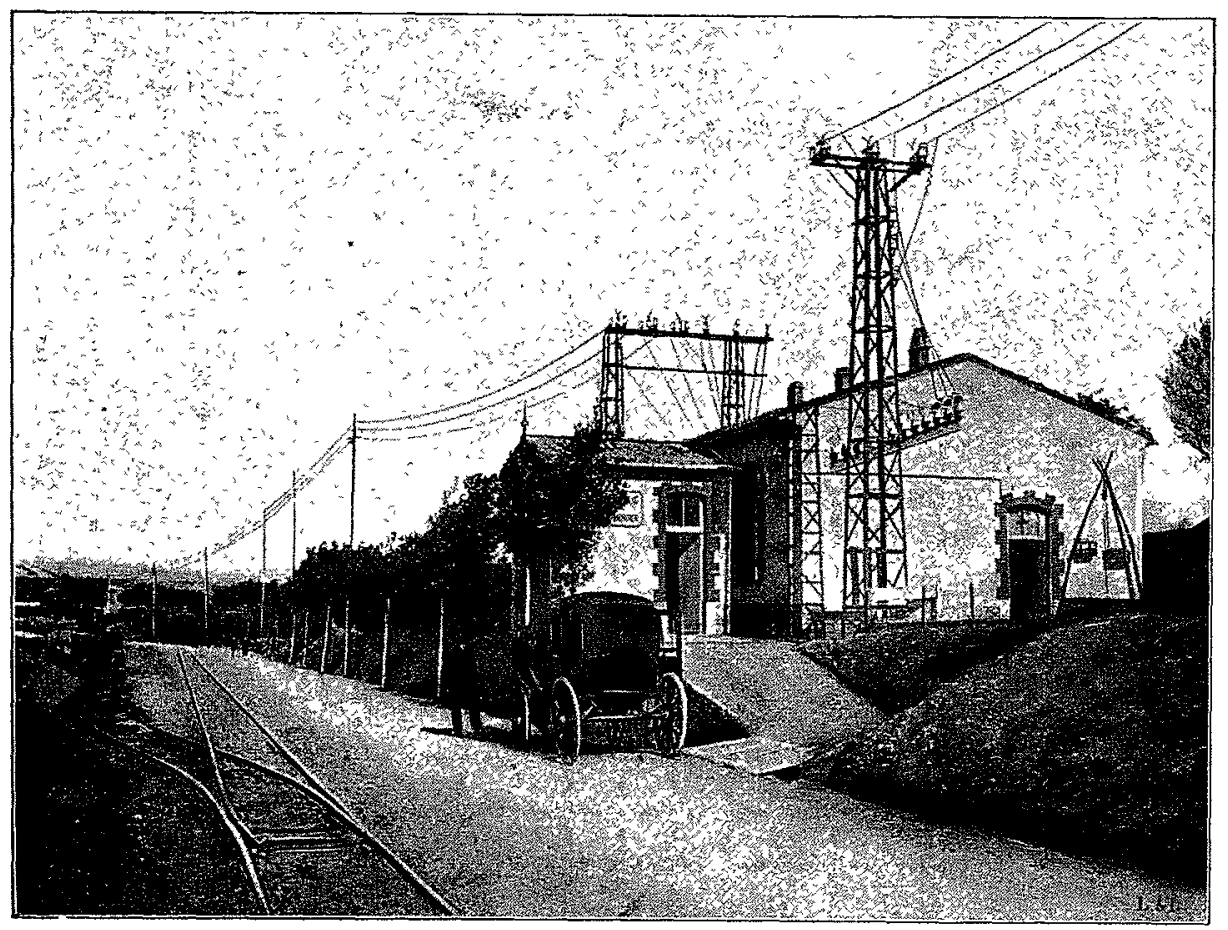

Fig. 4. - Vue du Poste central de distribution à Fabrezan. Arrivée et départ des lignes haute tension.

Il était intéressant de connaitre quelle serait l'influence de limpédance sur une aussi longue ligne.

On trouve l'inductance apparente d'un fil dans les tables de M. Blondel. En supposant l'écartement des fils de om6o, elle est de 1,07 milli-henry par kilomètre. La résistance d'un kilomètre de fil étant de $0,524 \mathrm{ohm}$, le facteur de réactance, c'est-à-dire le rapport de la réactance à la résistance ohmique, était de $0,64 \mathrm{I}$ à la fréquence de 50 périodes par seconde.

Le facteur de survoltage, c'est-à-dire le facteur par lequel il faut multiplier la tension au départ pour tenir compte de l'effet de la réactance de la ligne, est donné par la formule :

$$
\sqrt{\mathrm{I}+2\left(p-p^{2}\right)(\cos \varphi+m \sin \varphi-\mathrm{I})+p^{2} m^{2}}
$$

$p$ désignant la perte relative de tension admise en pour 100 et $m$ le facteur de réactance. La valeur obtenue par cette formule est de I,03, c'est-à-dire que, par suite de l'inspédance, la tension au départ devra atteindre 20850 volts.
A diverses reprises, la capacité du feeder a manifesté son importance, et de tous les phénomènes pouvant se présenter dans les lignes de grandes longueurs, c'est de beaucoup le plus saillant.

Si on soumet la question au calcul, en supposant un condensateur unique placé en un point déterminé de la ligne, comme on l'admet ordinairement, on trouve que le courant de charge devrait être de 4,6 ampères environ pour la tension de 20000 volts. L'expérience a démontré que ce chiffre était complètement erroné, car on lit I0,2 ampères comme courant de charge, avec une tension de 17600 volts, à l'usine génératrice, la ligne étant ouverte. D'ailleurs, c'est seulement à vide que la question de la capacité joue un rôle : dès que la ligne se charge, les phénomènes dus à cette propriété sont très atténués. Dans les conditions ci-dessus, le courant de charge correspond à plus de 3 oo kilo-volt. ampères, soit 72 ampères à la tension de 2480 volts sur la génératrice. Ce courant est nettement décalé en avant, car il suffit de 6 ampères d'excitation pour faire donner la tension de 2 t8o volts à l'alternateur, alors que sans la ligne il faudrait au moins $\mathrm{r} 2$ ampères : c'est donc le courant de charge de la ligne qui augmente l'aimantation des pièces polaires. On a vérifé que le courant de charge étalt bien proportionnel à la tension et à la fréquence; enfin, on a constaté que la tension à l'usine étant de I 7600 volts elle était à l'extrémité du feeder de 18300 volts, donc 700 volts plus élevée qu'à l'usine génératrice.

On a de plus constaté, en sectionnant le feeder et en ajoutant ensuite une autre ligne ayant 30 kilomètres, que le courant de charge va en augmentant jusqu’à 72 ampères (mesurés à la tension des alternateurs) pour une longueur de 70 kilomètres, puis qu'il diminue jusqu'à 38 ampères pour une distance de roo. kilomètres. Une autre dérivation de 35 kilomètres ajoutée au centre de distribution ne modifie plus l'intensité du courant de charge.

Voici les chiffres constatés, pour une tension de 2800 volts :

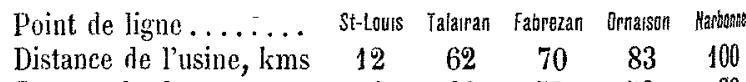
Courant de charge, amps $\begin{array}{llllll}0 & 68 & 72 & 52 & 38\end{array}$

Tous ces résultats peuvent être vérifiés par le calcul en appliquant aux lignes les formules relatives à la capacité uniformément répartie, malheureusement ces formules sort d'une complexité telle qu'il est difficile de les appliquer en pratique et de calculer à priori l'intensité du courant de charge et la surélévation de tension à vide. Heureusement, d'ailleurs, que tous ces phénomènes n'ont plus d'importance en charge et que leur influence ne se fait pas sentir; de sorte qu'à des distances de 100 kilo. mètres et la fréquence de 50 périodes on peut n'en tenir aucun compte dans la pratique. Ils servent à expliquer les surélévations de tension qui se produisent, notamment lors de la fusion des plombs; et à ce point de vue il est bon d'employer à l'usine génératrice des fusibles de très fortes sections ne fondant qu'à une intensité triple ou quadruple de l'intensité normale. Les fusibles employés dans toute l'installation sont des fils d'argent ou d'aluminium; ces. deux métaux ont donné l'un et l'autre de bons résultats.

Dans tout ce réseau qui présente, ainsi qu'il a été exposét' plus haut, une capacité dont l'importance n'est pas négli- 
geable, on n'a jamais constaté de phénomène de résonance, malgré la présence d'un troisième harmonique, dont l'im. portance est considérable dans les courbes de force électromotrice et de courant des alternateurs employés.

Le feeder est formé de trois câbles de $38 \mathrm{~m} / \mathrm{m}^{2}$ de section placés en triangle équilatéral et écartés de 60 centimètres. La flèche est de o m. 6o. Les poteaux ont io mètres de hauteur et sont espacés de 40 mètres; ils ne sont pas sulfatés mais bien enduits de carbonyle bouillant. Des isolateurs triple cloche de grande dimension ont été placés sur les poteaux. Tous ces isolateurs ont été essayés avant la pose à au centre de distribution. Les fils téléphoniques sont croisés tous les dix poteaux de façon à annuler les effets d'induction. S'il n'y a pas de fuites sur la ligne haute tension, la ligne téléphonique fonctionne parfaitement et la seule précaution à prendre consiste en la bonne isolation de la ligne haute tension. Les effets d'induction sont absolument négligeables et on n'entend aucune friture dans le téléphone si on a soin d'éloigner de I centimètre le récepteur de l'oreille.

Pour la surveillance du feeder qui traverse une contrée extrêmement montagneuse on a placé, tous les dix kilomètres, un cantonnier dont la mission est de faire une tournée tous les jours sur la ligne. Il se rend compte de l'état des isola-

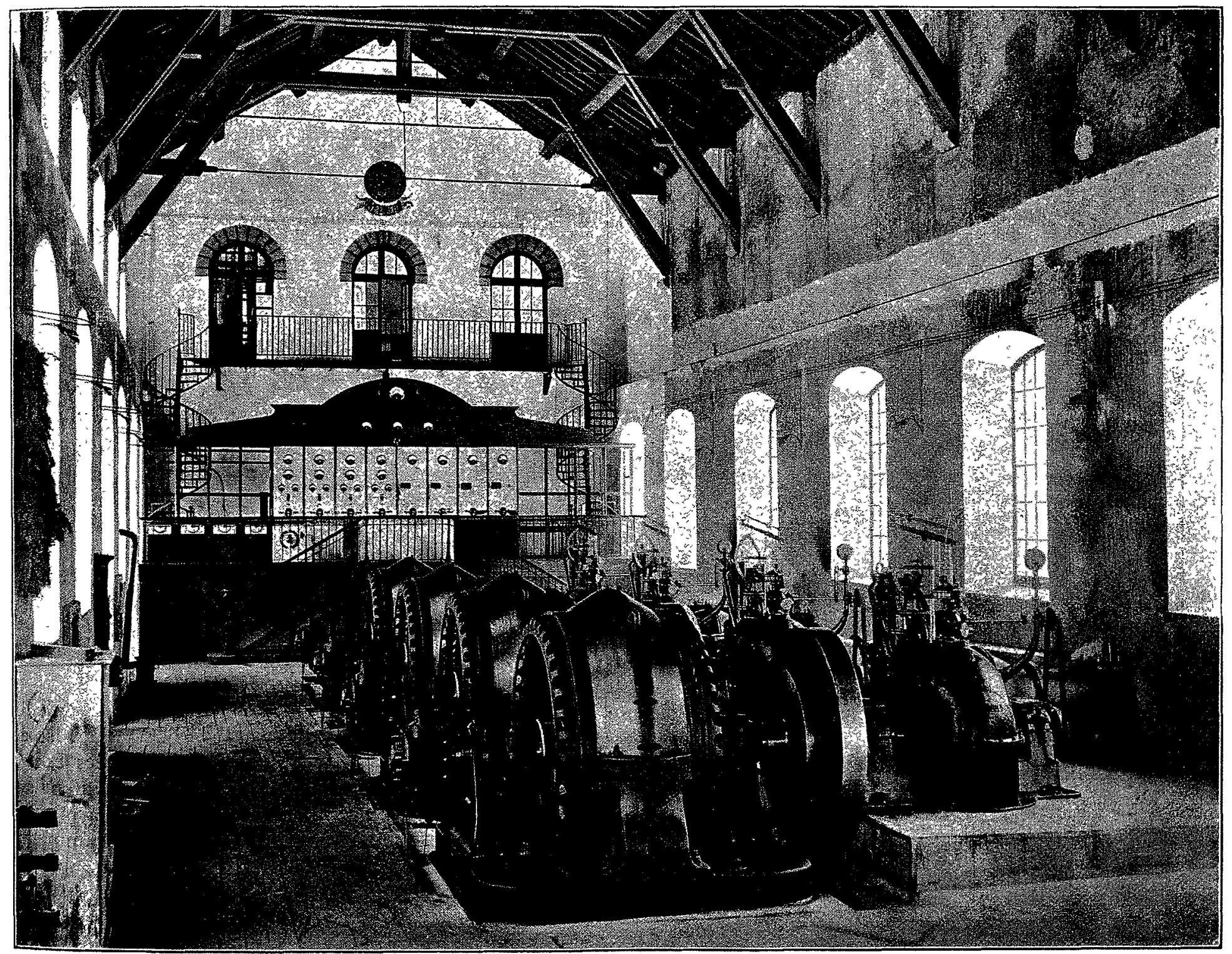

Fig. 5. - Vue générale des turbines et des alternateurs.

40000 volts et malgré cela quelques-uns furent percés au bout d'un certain temps par le courant; on reconnaissait facilement les isolateurs de qualité douteuse, parce qu'ils étaient lumineux la nuit. Les conducteurs eux-mêmes ne sont pas lumineux.

Pour bien s'assurer de l'isolation des lignes, on a fait fonctionner l'installation pendant une heure à la tension de 22000 volts, successivement avec chacun des fils à la terre, et à la tension de 30 ooo volts entre fils pendant trois heures.

Une ligne téléphonique placée sur des isolateurs double cloche, sur les mêmes poteaux que la ligne haute tension et à deux mètres en dessous de celle-ci, relie l'usine génératrice teurs, des poteaux et des fils. Ces cantonniers possèdent eux-mêmes un poste téléphonique sur la ligne reliant l'usine génératrice au centre de distribution et ils peuvent ainsi communiquer ce qu'ils ont vu dans leur tournée.

Le courant est supprimé tous les dimanches de 2 heures du matin à 3 heures de l'après-midi, et l'on fait pendant ce temps les réparations les plus urgentes.

Quelques court-circuits accidentels ont été occasionnés par des branches d'arbres, par le vent et aussi par des oiseaux de proie au début. Un grand-duc a été foudroyé par le courant et a été retrouvé en dessous des fils; plusieurs aigles, dont un avait $2 \mathrm{~m}$. 60 d'envergure, ont eu le même sort. 
Lorsqu'il y a court-circuit, il y a une surélévation de tension instantanée, qui dans un cas a atteint 35000 volts. Les parafoudres s'amorcent toujours à ce moment.

Le vent est très violent dans la région, et le parcours du feeder très capricieux : il part en effet de la cote $4^{30}$ pour s'élever à I ooo mètres dans la forêt des Fanges, passer au pied du pic de Bugarach et se maintenir à une altitude moyenne de 800 mètres pour aboutir dans la plaine de Fabrezan à la cote 200. L'hiver, les isolateurs ont été complètement recouverts de neige et ne formaient en certains endroits qu'un bloc de glace avec le poteau, cela n'empêchait pas l'installation de très bien fonctionner. L'isolation du feeder, mesurée au galvanomètre, étant en moyenne de 500000 ohms pour les trois fils et la terre; mais en temps de pluie ou de brouillard cette isolation tombe à 100000 ohms.

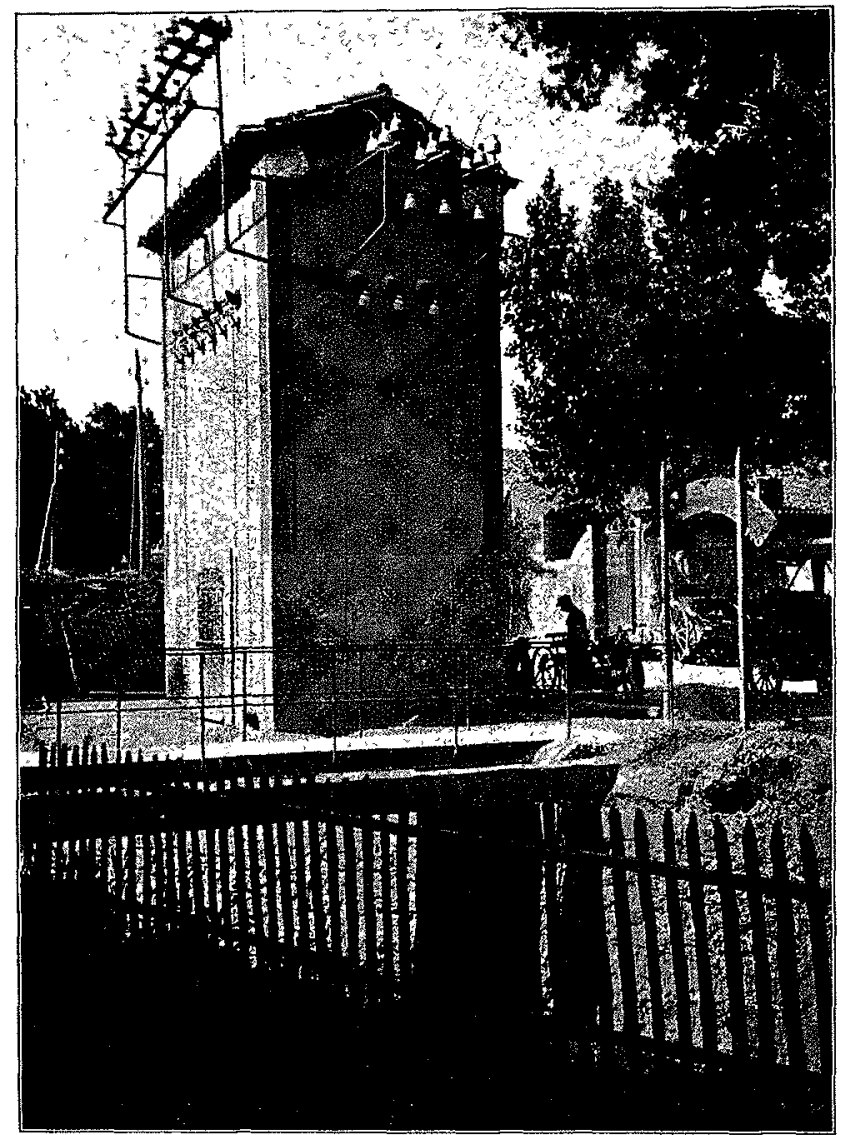

Fig. 6. - Vue d'un poste secondare de transformation $17000 / 5500$ volts.

Le poste central de distribution est situé à Fabrezan, c'est de ce point situé à 70 kilomètres de l'usine génératrice que partent les diverses dérivations.

Il comprend un grand tableau d'arrivée et de départ avec interrupteurs, coupe-circuits, ampèremètres et voltmètres. Ce tableau est exclusivement construit en fer; toutes les connexions sont faites en fil nu monté sur des isolateurs identiques à ceux de la ligne : les appareils de mesure sont également montés sur isolateurs. Les parafoudres à cornes sont placés à l'extérieur et leur ligne de terre est munie de résistances, toutefois il eut été préférable de les abriter. La tension est donnée à chaque instant dans ce poste par les lectures de volmètres branchés sur le secondaire de transformateurs témoins, très soigneusement isolés mais placés dans l'air; la construction de transformateurs aussi petits présente de grandes difficultés et ce n'est que grâce à une isolation exceptionnelle que l'on a pu rendre pratique le fonctionnement de semblables appareils.
C'est au poste central que se tient le chef de réseau qui peut communiquer par téléphone avec l'usine génératrice et fournir les renseignements nécessaires sur la tension à maintenir à cette usine.

Les dérivations partant du poste central sont au nombre de trois. L'une va à Narbonne, 30 kilomètres, l'autre à Carcassonne, 35 kilomètres, la troisième, appelée ligne du littoral, s'étend jusqu'à La Nouvelle. Ces dérivations sont toutes à 17000 volts, et la perte consentie en ligne est au maximum de 5 pour ioo.

Les villages qui se trouvent sur le passage de ces dériva. tions sont alimentés directement au moyen de transformateurs abaissant la tension de I 7000 à $\mathrm{i} 30$ volts, mais pour ceux qui ne sont pas sur le passage immédiat, pour ne pas augmenter indéfiniment le réseau à haute tension, on a eu soin d'abaisser auparavant la tension de 17 ooo a 5500 volts, et c'est avec cette dernière tension que de nouvelles lignes rayonnent tout autour des postes secondaires de distribution.

Ces postes secondaires de distribution sont composés de transformateurs dont la puissance est généralement de Ioo kilowatts, logés dans des kiosques spéciaux dans lesquels se trouvent encore des interrupteurs haute tension, des coupe-circuits et des parafoudres, aussi bien pour la haute que pour la basse tension. Ces kiosques contiennent généralement en plus un transformateur abaissant la tension. de 50000 à 125 volts pour la localité dans laquelle est construit le poste.

En établissant ces postes secondaires de distribution, on a réduit la surveillance de l'isolation du réseau haute tension, isolation qui est beaucoup plus difficile à maintenir et demande plus de soins que celle du réseau à 5000 volts. De plus, les difficultés de construction des transformateurs de puissance relativement faible pour la haute tension nécessitaient pour ainsi dire cette transformation.

Au nombre des détails intéressants de ces lignes à haute tension, il faut signaler les traversées de chemin de fer. Les lignes à 20000 volts traversent en quatre points les voies fer. rées, la Compagnie du Midi ne les a autorisées qu'à la condition de passer sous les ouvrages d'art et en câble isolé. On a donc exécuté ces passages en câbles sous plomb isolés au papier et raccordés aux lignes aériennes par des boîtes de jonction spéciales; ces câbles sont posés directement sur la maçonnerie de l'ouvrage d'art et le plomb de l'armature est relié au sol. Ces traversées, qui constituent somme toute un point faible des lignes, fonctionnent néanmoins d'une manière très satisfaisante.

Les dérivations à basse tension comprennent l'ensemble des lignes à 5000 volts... Ces_.lignes ne présentent d'autres différences avec les lignes à 20000 volts que l'emploi d'isolateurs de modèle plus réduit et une distance un peu plus faible entre fils. Chaque dérivation est protégée par dés coupe-circuits aériens à cornes.

Les postes de distribution dans chaque village contien: nent un transformateur à 5000 volts abaissant cette tension à i 25 volts. On n'a pas prévu d'interrupteurs haute tellsion, ce sont les coupe-circuits qui servent à cet effet : off peut couper sans inconvénient le courant de ces transformateurs en levant les barrettes mobiles de ces coupe-circuits modèle S. A. I. Un tableau basse tension sur marbre avet. interrupteurs et coupe-circuits commande la distribution de force motrice et d'éclairage du village. Généralementla puissance de ces transformateurs installés dans un village varie de 10 à 20 kilowatts. 
Les transformateurs 5 ooo volts sont également protégés par des parafoudres à cornes placés à l'extérieur des postes.

Les kiosques de transformateurs sont en maçonnerie et on a évité dans leur construction toute matière susceptible de s'enflammer.

\section{ORGANISATION DE L'EXPLOITATION}

Il est intéressant d'exposer comment on peut effectuer l'entretien et assurer l'exploitation d'un réseau aussi important, et cela à peu de frais. Car l'étendue des lignes constitue le point le plus faible d'une semblable installation. chef de l'exploitation qui a sous ses ordres directs un chef de réseau résidant au poste central de Fabrezan, d'où il peut communiquer par le téléphone particulier avec l'usine génératrice et par le téléphone de l'Etat ou le télégraphe avec les divers autres points du réseau. Le chef de réseau a pour mission spéciale la surveillance du feeder qui traverse une contrée montagneuse, très exposée au vent, et qui sur 30 kılomètres ne suit pas les routes : pour l'aider dans cette tâche, on a placé huit cantonniers sur le parcours du feeder, dont le rôle est uniquement de surveiller cette ligne et de faire des tournées tous les jours.

Les dérivations à haute tension sont sous la surveillance

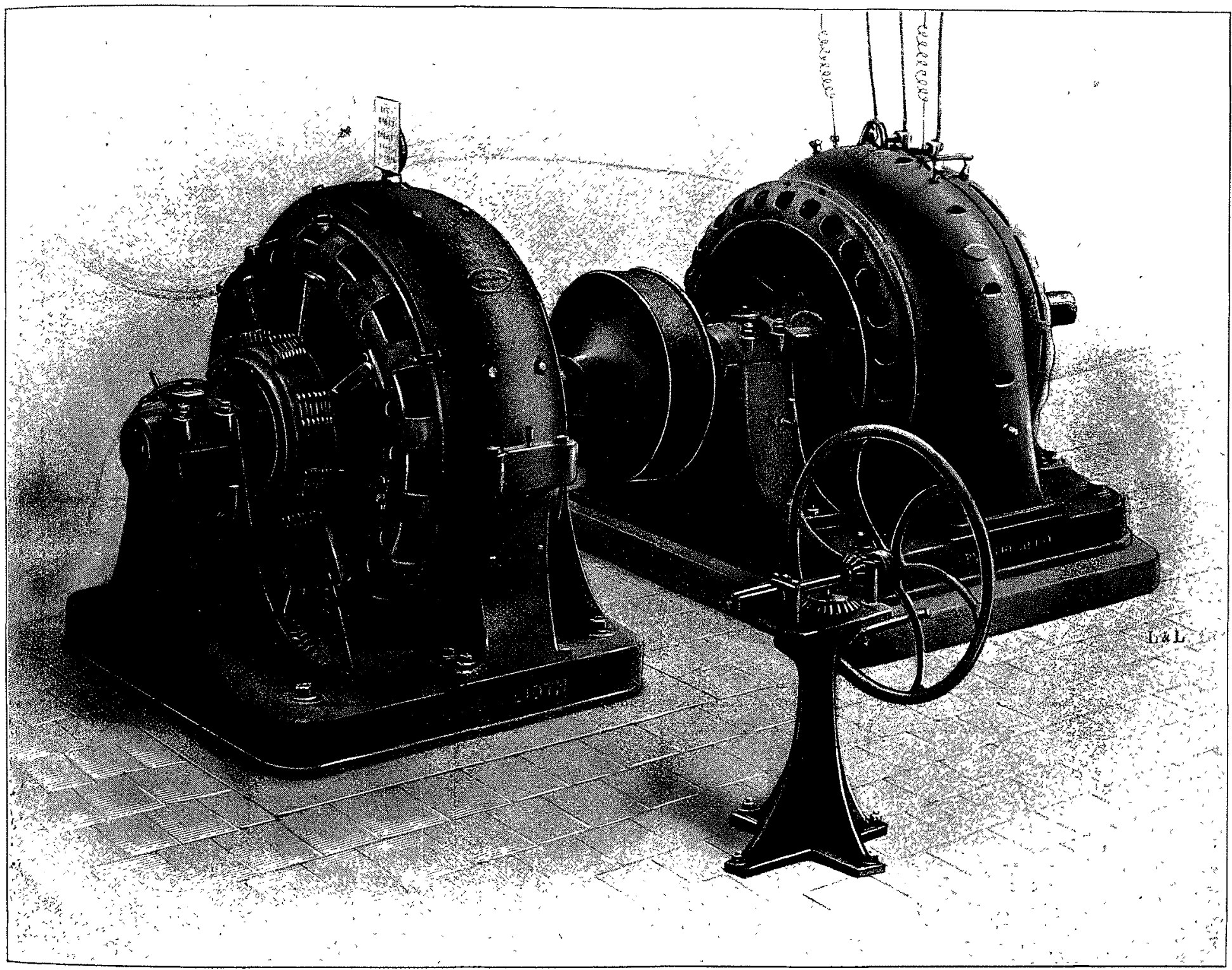

Fig 7. - Vue d'un groupe de transformation de courant triphasé en courant continu, x 50 kilowatts 300 volts 430 tours.

Au point de vue de l'organisation, celle adoptée pour l'exploitation du transport d'énergie de Saint-Georges par M. Estrade, le directeur de la Société méridionale de Transport de force, est en tous points remarquable.

Un véritable plan de mobilisation a été prévu et une copie se trouve entre les mains de chaque agent responsable. Ce plan détermine les fonctions de chacun, les rapports des divers agents entre eux, et prévoit ce qu'il y a à faire en cas d'accident pour le réparer et faire durer l'interruption du courant aussi peu de temps que possible. Un nombre considérable de cas ont été prévus.

Le personnel est placé sous la direcrion d'un ingénieur de deux sous-chefs de réseau qui ont également des cantonniers sous leurs ordres, mais en nombre bien inférieur à ceux du feeder, car les dérivations suivent les routes et il est aisé à bicyclette ou en automobile de se transporter d'un point à un autre.

Les sous-stations de Carcassonne et de Narbonne ont chacune un chef de grand poste qui s'occupe du matériel de ces sous-stations et des lignes autour de ces villes.

Enfin, il y a les chefs de poste qui se trouvent dans chaque village.

La plupart de ces agents s'occupent également de la partie commerciale et de l'exploitation des lignes à 5 ooo et 
I 25 volts et, par une correspondance bien établie et bien comprise, mettent rapidement la direction au courant des divers incidents de l'exploitation.

Tout cet ensemble fonctionne parfaitement et relativement à peu de frais.

Dans les deux villes de Carcassonne et de Narbonne, et en dehors de la distribution à courant triphasé 5 ooo volts pour les faubourgs et les environs de ces villes, il existe des distributions a courant continu trois fils avec une tension maximum de 300 volts entre les conducteurs extrêmes. Ces distributions étaient alimentées par des génératrices à courant continu, mues par des moteurs à vapeur ou hydrauliques. On a profité du courant triphasé venant de SaintGeorges pour installer des groupes de transformation en courant continu.

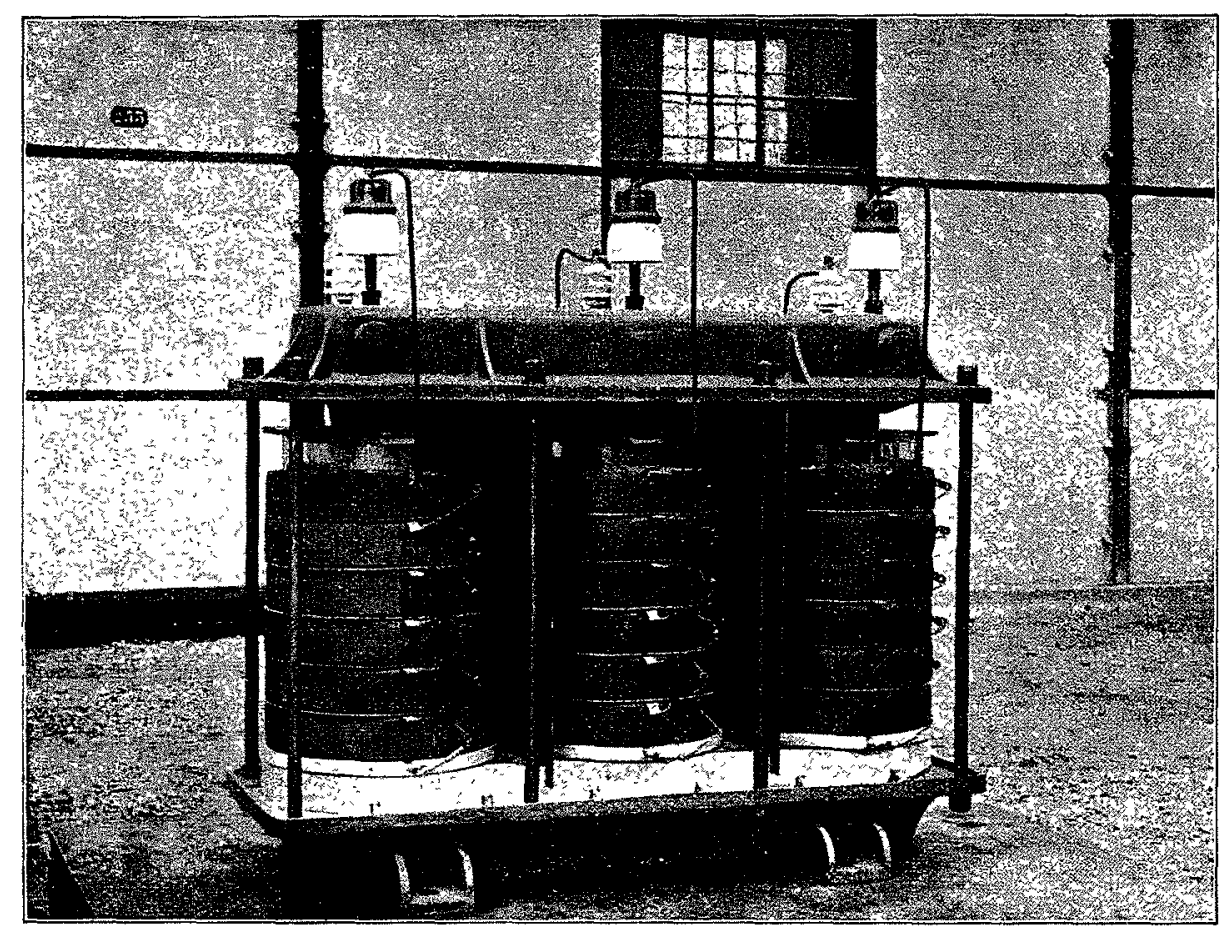

Fig. 8. - Vue dun transformateur triphasé 46 kilowatts $1,000 / 5000$ volts. charge en agissant sur l'excitation des génératrices à cov. rant continu, et le court-circuit passé, on recharge de suitte le moteur en agissant en sens inverse sur l'excitation des machines courant continu.

D'ailleurs, les court-circuits sont assez rares (même ceup produits par les coups de foudre), le décrochage ne se pro. duit pas souvent et ne présente pas une importance capi. tale, car les génératrices à courant continu marchent en parallèle avec des batteries ou avec des génératrices mues par des moteurs à vapeur ou hydrauliques pendant les. heures de plein service.

On peut se demander si des moteurs asynchrones n'eus: sent pas été préférables aux moteurs synchrones; les chan: ces de décrochage, évidemment, n'auraient pas été dimi nuées, mais une fois décrochés ils peuvent se remettre d'eux-mêmes en synchronisme, ce qui n'est guère possible avec des moteurs synchrones présentant comme il convient, peu de self-induction, demandant une excitation constante quelle que soit la charge, et par conséquent incapar bles de fonctionner comme moteurs asyn. chrones. La question est très discutable, mais dans le cas actuel, la raison du choix de ces moteurs a été, en les surexcitant, la possibilité de relever la tension en bout de ligne et par conséquent la possibilité de régler dans dè certaines limites la tension sur des dérivations aussi longues. Jusqu'à présent, d'ailleurs, le besoin de l'intervention des moteurs synchro. nes pour cette surélévation ne s'est pas falit sentir.

A Narbonne se trouvent deux groupes de transformation de 150 kilowatts chacun, composé de deux moteurs synchrones Alioth, de 225 chevaux 500 volts, accouplés directement à des génératrices courant continu à 300 volts tournant à 430 tours, et également du type Alioth.

A Carcassonne il existe un groupe identique à ceux de Narbonne.

Les moteurs électriques du réseau sont du

Ces groupes sont alimentés chacun par un transformateur triphasé de 200 kilowatts. Ces transformateurs sont isolés très soigneusement, mais, comme tous les transformateurs du réseau, ils ne sont pas plongés dans l'huile. Pour la mise en route des groupes, on amène les moteurs alternatifs au synchronisme en faisant fonctionner la génératrice à courant continu comme moteur, et en couplant à la manière ordinaire. Lorsque la ligne n'est pas chargée au moment de l'accrochage du premier moteur, il y a une surélévation de tension. Cette surélévation de tension atteint jusqu'à 25 ooo volts, elle s'évite d'ailleurs très facilement en pratique.

S'il y a un court-circuit sur la ligne, les moteurs synchrones peuvent se décrocher, à moins qu'on ne les décharge; le danger dépend d'ailleurs de l'intensité et de la durée du court-circuit. C'est ainsi qu'un court-circuit causé par une branche d'arbre ou un oiseau ne suffit pas pour produire le décrochage, les coups de foudre eux-mêmes ne le produisent pas toujours. Les chances de décrochage sont d'ailleurs bien diminuées par l'habileté plus ou moins grande de l'électricien au tableau. S'il s'aperçoit que la tension baisse par suite d'un court-circuit sur la ligne, il diminue la modèle asynchrone à courant triphasé, sauf dans les villes de Carcassonne et de Narbonne. La mise en marche s'effect tue en intercalant au moment du démarrage une résistancet dans l'induit du moteur. Cette résistance pour les petits moteurs est fixée sur l'une des faces de l'induit, fait corps et tourne avec lui.Une fois la vitesse du synchronisme atteinte cette résistance est mise en court-circuit au moyen d'une pièce de bronze que l'on manœuvre au moyen d'une tige traversant l'arbre du moteur, qui est foré à cet effet et muni d'un bouton à son extrémité.

Lorsque les moteurs doivent démarrer avec une charge pourrait être logée sur l'induit et est extérieure : dans't cas le moteur est muni de bagues et de frotteurs ef charbon.

Dans les̄ villes de Carcassonne et de Narbonne les mot? teurs ont été placés généralement sur le couranít continuel fonctionnent à la tension de 250 volts.

La partie financière de cette affaire n'est pas moints remarquable que les diverses autres parties de cette entrte prise. élevée, la résistance, de dimension trop considérable, nte 
Elle est basée sur un principe nouveau qui, d'après les termes mêmes de M. Blondel, rapporteur de la commission des distributions d'électricité, "constitue un procédé ingénieux pour vulgariser les installations d'éclairage municipal ». Grâce, en effet, à des combinaisons particulières, la Société méridionale de Transport de force a pu se contenter de construire à ses frais l'usine génératrice et les tignes à haute tension. Tout le reste a été fait aux frais des communes et des particuliers.

L'éclairage municipal est assuré gratuitement à raison de 25 lampes à incandescence de 16 bougies par mille habitants. L'éclairage des particuliers est fait à forfait; la lampe de 16 bougies est vendue 32 francs par an; il n'y a pour ainsi dire pas de compteurs, sauf à Carcassonne et à Narbonne, mais par contre il y a des basculateurs Estrade qui, dansles installations de plusieurs lampes, ne permettent pas d'en avoir plus d'allumées à la fois que cela est prévu par la police de l'installation.

Une grande partie de l'énergie est vendue comme force imotrice, mais en principe les moteurs doivent s'arrêter aux heures où commence l'éclairage. Ces moteurs sont bealicoup urilisés pour des élévations d'eau; généralement chaque commune a remplacé le moteur à pétrole qu'elle possédait pour cet usage par un moteur électrique. Il y a encore une foule de moteurs pour des arrosages, des inanutentions de vins, des brasseries, des fabriques dè glace et autres industries similaires de la région. Tous ces moteurs sont des moteurs triphasés asynchrones Alioth; ils sont généralement munis d'un disjoncteur a minima qui, dans le cas où le courant viendrait à manquer momentanément, ouvrirait le circuit et empêcherait, lorsque le courant est rétabli, le moteur de démarrer sans résistance et par conséquent éviterait la détérioration de l'apparenl.

Les recettes réalisées dès le début ont été considérables; elles couvrent non seulement les frais d'exploitation, mais encore elles ont permis d'assurer dès la seconde année une rémunération convenable au capital. D'ailleurs ces installations ne se bornent pas au département de l'Aude, mais gagnent l'Hérault dans la direction de Béziers.

L. P.

\section{ÉTUDE SUR LES}

\section{BARRAGES EN MAÇONNERIE \\ et Murs de Réservoirs (suite)}

\section{DIVERS MODES DE CONSTRUCTION DES BARRAGES}

Les barrages, ou plus généralement les murs ayant à supporter une charge d'eau, peuvent être classés en trois catégories, suivant la nature des matériaux qui les composent : digues en terre, barrages mixtes et barrages en maçonneries.

Les barrages proprement dits peuvent encore ètre divisés en barrages fixes et barrages mobiles. Nous ne nous occuperons, dans cet article, que des ouvrages fixes; les barrages mobiles n'étant guère employés que par le service de la navigation et ne créant d'ailleurs que des retenues de hible hauteur.

\footnotetext{
Digues en terre. - L'emploi des digues en terre remonte à la plus haute antiquité. Aux Indes, notamment, on trouve nncore des digues dont la construction est très ancienne et
}

dont les dimensions sont considérables, la longueur de quelques-unes atteignant jusqu'à 15 kilomètres et mème plus. Parmi les digues anciennes les plus remarquables on peut citer celle de l'étang de Ponairy, district de Trichnapoly, province de Madras, qui avait plus de 48 kilomètres de longueur.

Une digue en terre, plus encore qu'un barrage en maçonnerie, doit être parfaitement étanche, car, si elle ne remplissait pas cette condition, elle donnerait lieu à des infiltrations appréciables qui, allant en s'amplifiant de plus en plus, ne tarderaient pas à créer une ou plusieurs brèches et finalement la ruine de l'ouvrage avec toutes ses désastreuses conséquences.

Pour réaliser cette étanchéité, les digues en terre doivent ètre constituées par un bon corroi, e'est-à-dire par une substance homogène et imperméable. Il faut éviter d'employer des terres trop argileuses, car elles sont trop glissantes, ainsi que des terres sab!onneuses qui sont trop perméables. Le meilleur corroi parait être une terre argilo-sableuse dans laquelle l'argile est juste suffisante pour agglomérer entre eux les grains de sable. Dans la digue du réservoir de Mittersheim (canal des houillères de la Sarre), le corroi est composé par moitié de sable et d'argile. Quelquefois l'on ajoute au corroi une petite quantité de chaux hydraulique soit à l'état de poudre, soit sous forme de lait de chaux. Il faut de plus pilonner soigneusement et fortement en procédant par couches de faible épaisseur.

Les digues françaises sunt constituées par un remblai homogène. Leur parement amont est généralement incliné á 3 de base pour 2 de hauteur, il est tantôt rectiligne et

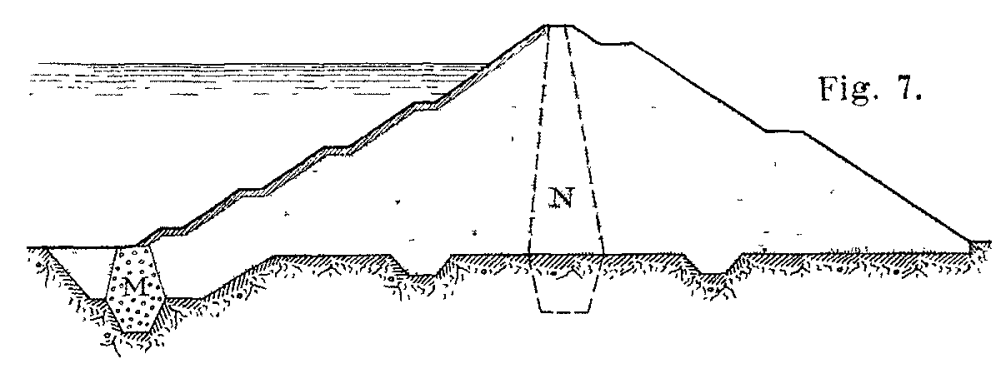

tantôt entrecoupé de gradins horizontaux. Le parement a val est le plus souvent incline suivant la direction naturelle des terres employées. Pour protéger le parement amont contre l'action destructive des vagues, celui-ci est habituellement muni d'un revêtement de maçonnerie à mortier; on peut aussi constituer ce revêtement par des enrochements reposant sur une dalle en mortier, savaté ou armé, de faible épaisseur.

Pour arrêter les infiltrations à la base on dispose, au pied amont de la digue, un mur de garde $M$, généralement en béton, que l'on descend jusqu'à ce qu'on ait trouvé une couche de terrain imperméable. Ce mur est flanqué, á l'amont et à l'aval, de deux massifs de terre fortement corroyée, celui d'aval faisant corps avec le massif général de la digue. De plus, la base de la digue n'est pas rectiligne, mais disposée suivant une ligne brisée.

Pour surélever le niveau du lac Orédon, qui se trouve dans les Pyrénées à 1850 mètres d'altitude et qui sert à l'alimentation du canal de la Neste, on a construit un barrage en terre. Pour éviter les infiltrations, on a constitue le parement amont par une première couche de béton de 0in 20 d'épaisseur reposant sur un perré de pierres sèches. Sur ce béton on a disposé un second perré formant 